\title{
Clinical Study \\ Impact of Exogenous Gonadotropin Stimulation on Circulatory and Follicular Fluid Cytokine Profiles
}

\author{
N. Ellissa Baskind, ${ }^{1}$ Nicolas M. Orsi, ${ }^{2}$ and Vinay Sharma ${ }^{1}$ \\ ${ }^{1}$ The Leeds Centre for Reproductive Medicine, Leeds Teaching Hospitals NHS Trust, Seacroft Hospital, \\ York Road, LS14 6UH Leeds, UK \\ ${ }^{2}$ Women's Health Research Group, Leeds Institute of Cancer \& Pathology, St James's University Hospital, \\ Wellcome Trust Brenner Building, Beckett Street, LS9 7TF Leeds, UK
}

Correspondence should be addressed to N. Ellissa Baskind; ellissa@doctors.org.uk

Received 23 September 2014; Accepted 11 November 2014; Published 30 November 2014

Academic Editor: Stefania A. Nottola

Copyright (C) 2014 N. Ellissa Baskind et al. This is an open access article distributed under the Creative Commons Attribution License, which permits unrestricted use, distribution, and reproduction in any medium, provided the original work is properly cited.

\begin{abstract}
Background. The natural cycle is the prototype to which we aspire to emulate in assisted reproduction techniques. Increasing evidence is emerging that controlled ovarian hyperstimulation $(\mathrm{COH})$ with exogenous gonadotropins may be detrimental to oogenesis, embryo quality, and endometrial receptivity. This research aimed at assessing the impact of $\mathrm{COH}$ on the intrafollicular milieu by comparing follicular fluid (FF) cytokine profiles during stimulated in vitro fertilization (IVF) and modified natural cycle (MNC) IVF. Methods. Ten women undergoing COH IVF and 10 matched women undergoing MNC IVF were recruited for this pilot study. 40 FF cytokine concentrations from individual follicles and plasma were measured by fluid-phase multiplex immunoassay. Demographic/cycle/cytokine data were compared and correlations between cytokines were computed. Results. No significant differences were found between $\mathrm{COH}$ and $\mathrm{MNC}$ groups for patient and cycle demographics, including outcome. Overall mean FF cytokine levels were higher in the MNC group for 29/40 cytokines, significantly so for leukaemia inhibitory factor and stromal cell-derived factor-1 $\alpha$. Furthermore, FF MNC cytokine correlations were significantly stronger than for $\mathrm{COH}$ data. Conclusions. These findings suggest that $\mathrm{COH}$ perturbs intrafollicular cytokine networks, in terms of both cytokine levels and their interrelationships. This may impact oocyte maturation/fertilization and embryo developmental competence.
\end{abstract}

\section{Introduction}

Controlled ovarian hyperstimulation $(\mathrm{COH})$ with gonadotropins has improved success rates of in vitro fertilization (IVF) by increasing the number and opportunity for selection of embryos before transfer [1-3] as well as permitting the cryopreservation of supernumerary embryos for further fertility treatment $[4,5]$. The basis of $\mathrm{COH}$ is to support the growth of multiple follicles to the preovulatory stage, a process achieved by bypassing physiological regulatory mechanisms. Urinaryderived or recombinant follicle stimulating hormone (FSH) is administered to increase serum concentrations above the threshold required for dominant follicle selection, thus enabling the entire cohort of recruited follicles to develop and attain preovulatory status [4]. Luteinising hormone (LH) is often coadministered although, following pituitary downregulation, this is not essential for follicular development as remnant basal LH levels are sufficient to stimulate the theca cells. Administration of a GnRH analogue (long protocol) or an antagonist (short protocol) that desensitizes the pituitary is primarily used to prevent premature LH surge as a consequence of supraphysiological serum oestradiol $\left(\mathrm{E}_{2}\right)$ levels which, if it occurs, can lead to premature luteinisation and/or ovulation.

With few exceptions [6], the last two decades have witnessed a mounting body of evidence indicating that ovarian stimulation has a detrimental effect on oogenesis, embryo quality, and endometrial receptivity [7-13]. More specifically, Sharma et al. [7] and Pellicer et al. [14] demonstrated that retrieval of $>10$ oocytes per woman adversely affected their quality based on oocyte/embryo morphology, fertilization, and implantation rates. More recently van der Gaast 
et al. [15] found 13 oocytes to be the optimum number retrieved in order to achieve a pregnancy using a long protocol, above which there was a fall in pregnancy rates. An extreme example of the negative impact of $\mathrm{COH}$ is the excessively high number of poor quality oocytes seen in ovarian hyperstimulation syndrome (OHSS), which is putatively attributable to detrimental supraphysiological $\mathrm{E}_{2}$ levels [16]. These observations in humans are supported by a number of rodent studies that investigated the impact of exogenous gonadotropin stimulation on oocytes and demonstrated a delay in embryo development $[17,18]$. It has been suggested that gonadotropin stimulation may affect oocyte maturation and the completion of meiosis, thus leading to an increased risk of having aneuploid oocytes and/or embryos [10, 19]. As such, in vitro maturation (IVM) has been proposed as an alternative strategy since it reduces exposure to exogenous gonadotropin stimulation, but the process itself introduces a host of other variables/complications (e.g., disruption of the meiotic spindle) that do not allow a fair comparison of these approaches to be made [20]. von Wolff et al. [21] recently demonstrated a varying endocrine follicular milieu together with the concentration of putative markers of oocyte quality, specifically anti-Müllerian hormone (AMH) between $\mathrm{NC}$ and $\mathrm{COH} \mathrm{FF}$, and suggest that this may be the cause for the lower oocyte quality following $\mathrm{COH}$ compared with naturally matured oocytes.

There has also been some concern that suppressed LH concentrations in the late follicular phase may be detrimental through downstream perturbations in follicular steroid synthesis. Consequently, stimulation protocols incorporating exogenous LH were developed, resulting in an increase in the percentage of diploid and good quality embryos obtained $[22,23]$. By contrast, other investigators have reported a reduction in fertility and increased risk of miscarriage when incorporating exogenous LH into protocols $[24,25]$. Such contradictory findings support the notion of a "LH window" below which $\mathrm{E}_{2}$ production is inadequate and above which LH may begin premature luteinisation and be detrimental to follicular development [26]. von Wolff et al. [21] postulate that the reduced levels of intrafollicular AMH they demonstrated following $\mathrm{COH}$ compared with $\mathrm{NC}$ may be attributed to LH suppression, resulting initially in low follicular androgen concentrations, and subsequently to low AMH production which in turn may be responsible for lower oocyte quality.

Natural cycle IVF (NC-IVF) has been proposed as an alternative treatment for older women and poor responders [27]. Indeed, there has been a resurgence of interest in NCIVF for all patients in recent years because it avoids $\mathrm{COH}$ and its potential sequelae. Moreover, this also supports the international drive to reduce multiple pregnancies rates with elective single embryo transfer and to minimise complications such as OHSS [28-30]. Pelinck et al. [1] conducted a systematic review of 1,800 natural IVF cycles reported between 1989 and 2000 and concluded that NC-IVF has a pregnancy rate of less than $10 \%$ per cycle. More recent reports concur, presenting a similar $15.2 \%$ live birth rate per initiated cycle in all reported unstimulated NCs in women $<35$ years $(n=795)$ in the United States (2006-2007) [31].
A compromise between these approaches has been described: mild ovarian stimulation IVF. This method incorporates the use of low dose gonadotropin stimulation together with a gonadotropin releasing hormone $(\mathrm{GnRH})$ antagonist aimed at generating fewer than eight oocytes per cycle [32]. The term modified natural cycle IVF (MNC-IVF) is applied when drugs (e.g., human chorionic gonadotropin (hCG)) are used to induce final oocyte maturation whereby a $\mathrm{GnRH}$ antagonist is administered during a spontaneous cycle to reduce the risk of cancellation [33] and/or where luteal support is provided.

During folliculogenesis, follicular fluid (FF) composition exhibits dynamic changes as individual follicular cell types respond to gonadotropins by secreting different hormones and cytokines [34, 35]. As growth factors regulating all stages of folliculogenesis, cytokines have been shown to govern the development/function of somatic cells and the oocyte as well as the composition of FF [36-42]. Given that oocyte quality influences subsequent embryo viability [43], it has been suggested that the disruption in the balance of these intrafollicular mediators following $\mathrm{COH}$ may influence cycle outcome [44-52]. The correct regulation of cytokine networks is essential to support normal physiology and this central role is underscored by the fact that inflammatory/immune dysfunctions underpin many pathological reproductive conditions, resulting in both local and systemic changes in cytokine profiles [53-55].

Studies have measured individual FF cytokines throughout the menstrual cycle. For example, the levels of interleukin- (IL-) 8, a chemotactic and angiogenic cytokine essential to folliculogenesis, have been found to rise from the midfollicular to the late follicular phase. These levels are comparable to those found during a $\mathrm{COH}$ cycle [56], implying that granulosa cell (GC) and theca cell (TC) IL-8 secretion is a true physiological phenomenon associated with follicular growth/maturation rather than resulting from gonadotropin stimulation. In vitro enhancement of IL-8 secretion by cultured GCs and TCs was evident following exposure to IL- $1 \alpha$ and IL- $1 \beta$, but not tumour necrosis factor- (TNF-) $\alpha$, suggesting that IL- 8 is both gonadotropin and cytokine-induced and may thus be involved in the hormonally regulated stages of folliculogenesis and ovulation [57].

Although cytokines are readily detected in FF, the complexity of their network regulation makes their study in isolation difficult to interpret. In view of their biological properties (pleiotropism, synergy, antagonism, functional redundancy, and differential sensitivity) [58-60], cytokines should ideally be investigated in terms of their interrelationships as much as in terms of their absolute concentrations. Whilst a recent study by Bersinger et al. [61] failed to demonstrate a difference in $13 \mathrm{FF}$ cytokines between women undergoing $\mathrm{NC}$ and $\mathrm{COH}$ IVF, no specific attention was paid towards the complex interrelations within the cytokine networks. To date, there has been a paucity of studies focusing on minimal stimulation regimens and MNCs, and comparisons of isolated cytokine concentrations (e.g., vascular endothelial growth factor (VEGF) in $\mathrm{COH}$ and NC-IVF) have been inconsistent $[62,63]$. Exogenous gonadotropins may disrupt intrafollicular cytokine networks, in turn affecting oocyte 
developmental potential. Therefore, this pilot study aimed at examining the impact of gonadotropins on the intrafollicular cytokine milieu in MNC and following $\mathrm{COH}$ cycles.

\section{Materials and Methods}

2.1. Patient Recruitment and Sample Collection. From November 2008 to March 2009, ten women who required treatment with IVF/ICSI due to unexplained or male factor infertility aged 25-35 years with a body mass index (BMI) 19-30 were selected to undergo MNC-IVF/intracytoplasmic sperm injection (ICSI) at the Assisted Conception Unit, St James's University Hospital, Leeds, UK. These patients were matched with ten women undergoing $\mathrm{COH}$-IVF/ICSI. Only nonsmokers and women who drank $<6$ units alcohol per week were included. They were required to be ovulatory (confirmed by transvaginal ultrasound scan (TVUSS), progesterone levels, or commercial LH surge kits within the preceding three months) and have a normal endocrine profile (early follicular phase $\mathrm{FSH}<8.0 \mathrm{IU} / \mathrm{L}$ and $\mathrm{E}_{2} 50-$ $200 \mathrm{pmol} / \mathrm{L}$ ), a negative infection screen (including negative serum Chlamydia antigens), and normal pelvic anatomy confirmed by TVUSS and laparoscopy. Furthermore, they were required to have no risk factors for pelvic pathology (e.g., history of pelvic inflammatory disease, incomplete miscarriage, ectopic pregnancy, cervical dyskaryosis, and abdominal/pelvic/cervical surgery). Women with coexisting morbidity (e.g., autoimmune diseases, inflammatory conditions, and diabetes mellitus) and those taking regular medications were also excluded. The study protocol was approved by National Research Ethics Service, Leeds (East) Research Ethics Committee, and all participants provided written informed consent.

All women underwent a baseline TVUSS (ALOKA SSSD $1700)$ in the early follicular phase. In the MNC cohort, a TVUSS assessment was performed on alternate days from day 8 of the cycle, until the mean maximal diameter (MMD) measured in two planes (sagittal and transverse) of the dominant follicle measured $\geq 14 \mathrm{~mm}$, after which they were performed daily. Women were asked to use urinary LH kits twice daily (06:00-08:00 and 18:00-20:00) in order to identify the onset of LH surge prior to spontaneous ovulation. An injection of 5,000 IU hCG (Pregnyl (Organon, Cambridge, $\mathrm{UK})$ ) was given when the MMD of the lead follicle measured $\geq 17 \mathrm{~mm}(17.0-18.1 \mathrm{~mm})$. If the urinary LH kit was positive, ultrasound directed oocyte retrieval (UDOR) was performed the day after the surge was detected; otherwise it was planned for $36 \mathrm{~h}$ after hCG. For subsequent analysis, women who had a spontaneous LH surge and women who were administered exogenous hCG were grouped together as the MNC cohort. All women in the $\mathrm{COH}$ arm underwent the long protocol. Pituitary downregulation was attained using leuprorelin acetate SR $3.75 \mathrm{mg}$ (Prostap (Wyeth, Maidenhead, Berkshire, UK)) administered on the first day of the menstrual cycle. $\mathrm{COH}$ was achieved with $225 \mathrm{IU}$ human menopausal gonadotropin (HMG) daily (Menopur, Ferring, Slough, Berkshire, UK). As with the MNC-IVF/ICSI cycle, when one or more follicles had an MMD of $\geq 17 \mathrm{~mm}, 5,000 \mathrm{IU}$
hCG (Pregnyl (Organon, Cambridge, UK)) was administered $36 \mathrm{~h}$ prior to UDOR.

All UDORs were performed between 09:00 and 11:00 to accommodate putative circadian variations in ovarian physiology. Whole blood was collected immediately before sedation in EDTA vacutainers. Dead space (containing $1.5 \mathrm{~mL}$ $0.9 \%$ sodium chloride solution) within the oocyte harvesting needle and tubing was constant/uniform throughout such that the first $1.5 \mathrm{~mL}$ aspirated was checked and discarded. Subsequent aspirate was considered to contain FF and, following oocyte assessment and retrieval, was subsequently labelled to ensure longitudinal tracking of the corresponding oocyte to its fate (for multifollicular cycles). Follicles were flushed up to four times with culture medium (Enhance HTF Culture Medium with HEPES; Conception Technologies, San Diego, California, USA) if no oocyte was obtained in the initial aspirate to minimise the risk of inadvertently aspirating a second follicle and collecting the oocyte from the previous one due to being contained within the dead space. All ultrasonically visible follicles were individually aspirated irrespective of their size. The aspiration pressure applied was uniform on all follicles $(183-185 \mathrm{~mm} / \mathrm{Hg})$.

All samples were immediately stored on ice. Whole blood and FF samples were centrifuged $\left(2,000 \mathrm{rpm}\right.$ at $4^{\circ} \mathrm{C}$ for 10 minutes) to isolate plasma and remove cell debris, respectively. All samples were frozen at $-80^{\circ} \mathrm{C}$ within one hour of retrieval until required for analysis. In the $\mathrm{MNC}$ cohort, only the FF from the single dominant follicle was analysed. In the $\mathrm{COH}$ cohort, FF analysis was performed on the follicles yielding the oocytes that generated transferred embryos (since double ETs were performed in these cycles, the follicle selected for analysis was the one yielding the embryo with the highest morphological grading).

In both cycles, routine procedures for fertilization with IVF/ICSI were performed as previously described [64]. Embryo transfer (ET) was performed 84-90 h after hCG injection. A single ET was performed in the MNC cohort, whilst a double ET was performed in the $\mathrm{COH}$ cohort (as per ACU protocols at the time). All women in the MNC cohort who underwent an ET received 2,500 IU hCG (Pregnyl) on the day of ET and again $72 \mathrm{~h}$ later for luteal support. Women in the $\mathrm{COH}$ cohort who developed $<15$ follicles had an identical luteal support regimen, whereas those women with $\geq 15$ follicles following $\mathrm{COH}$ were given daily intramuscular injection of $100 \mathrm{mg}$ progesterone (Gestone, Nordic Pharma, Reading, Berkshire, UK), which was continued throughout the first trimester of pregnancy. Pregnancy tests were performed on first void urine 14 days after ET with a commercial urinary kit. A clinical pregnancy was defined as one demonstrating a gestational sac with a fetal pole and a fetal heart or an ectopic pregnancy by TVUSS at 6-7 weeks' gestation.

2.2. Fluid-Phase Multiplex Immunoassay. Cytokine levels in both FF and plasma were measured by fluid-phase cytometric multiplex immunoassay (Bio-Rad Laboratories, Hercules, CA, USA) (Bio-rad assays: Human Cytokine 27plex Assay M50-0KCAF0Y; Human Cytokine 21-plex Assay MF0-005KMII) using a Luminex 100 cytometer (Luminex 
Corporation, Austin, Texas, USA) equipped with BioPlex 4.0 Manager software (Bio-Rad Laboratories, Ltd., Hertfordshire, UK), as previously described [65]. Target cytokines included interleukin- (IL-) 1 receptor antagonist (ra), IL-2ra, IL-3, IL-6, IL-7, IL-8, IL-9, IL-10, IL-12 (p40), IL-12 (p70), IL-13, IL-15, IL-16, IL-18, leukaemia inhibitory factor (LIF), granulocyte macrophage-colony stimulating factor (GM-CSF), macrophage- (M-) CSF, granulocyte- (G-) CSF, stem cell factor (SCF), interferon- (IFN-) $\alpha$, IFN- $\gamma$, IFN- $\gamma$ inducible protein- (IP-10), TNF- $\alpha$, TNF- $\beta$, TNF related apoptosis inducing ligand (TRAIL), VEGF, platelet derived growth factor (PDGF), basic fibroblast growth factor (b-FGF), nerve growth factor- (NGF-) $\beta$, stem cell growth factor- (SCGF) $\beta$, growth regulated oncogene- (GRO-) $\alpha$, macrophage inflammatory protein- (MIP-) $1 \beta$, monocyte chemoattractant protein- (MCP-) 1, MCP-3, eotaxin, regulated upon activation of normal $\mathrm{T}$ cell expressed and secreted (RANTES), stromal cell-derived factor- (SDF-) $1 \alpha$, cutaneous T-cell attracting chemokine (CTACK), monokine induced by IFN- $\gamma$ (MIG), and macrophage migration inhibitory factor (MIF).

2.3. Contamination and Standardisation. Oocyte retrieval frequently results in disruption of the intraovarian vasculature such that blood (macroscopic or microscopic) contaminates the FF retrieved [66]. Furthermore, although the needle and tubing were primed with normal saline at the commencement of aspiration of each ovary, in between subsequent follicular aspirations, protein-free flush medium formulated with gentamicin (enhanced HTF culture medium with HEPES; Conception Technologies, San Diego, California, USA) was used, with potential dilution of the FF [67]. Such contamination/dilution was accounted for in the FF cytokine analysis. This entailed cytokine, total protein (by Lowry assay, Bio-Rad), and von Willebrand factor (vWF; by enzyme-linked immunosorbent assay; R\&D Systems, Abingdon, UK) measurement in both plasma and FF. Since vWF is a large plasma multimeric glycoprotein that does not pass through the basement membrane and is not produced by follicular cells, it enabled accurate quantification of FF blood (and therefore circulatory cytokine) contamination (present authors, manuscript under review). The dilutional effect of the flush medium was instead accounted for by standardising both FF and plasma (the latter to enable a valid comparison with the former) cytokine concentrations to total protein (pg cytokine/mg protein).

2.4. Statistical Analysis. Chi-squared, independent samples $t$-tests, or Mann-Whitney $U$ tests were used to compare $\mathrm{MNC}$ and $\mathrm{COH}$ patient demographics, cycle details, FF, and plasma cytokines following tests for normal distribution by Shapiro-Wilk test (Stata/SE 11.1, Texas, USA). In order to address the interrelationships between multiple cytokines and the impact that $\mathrm{COH}$ has upon these, heat maps were generated using R 2.7.0 software (R Foundation for Statistical Computing, Vienna, Austria). Correlations between the different cytokines were determined for $\mathrm{MNC}$ and $\mathrm{COH}$ data using Kendall's tau as a measure of correlation (Stata/SE 11.1). Resulting $P$ values were adjusted for multiple comparisons with Holm's correction ( $P<0.05$ was considered significant).

\section{Results and Discussion}

3.1. Participant, MNC, and COH Cycle Demographics. In the MNC cohort, one patient had a positive LH surge and therefore did not receive exogenous hCG. At the time of UDOR 12 hours after the positive surge, spontaneous ovulation had occurred such that no FF was retrieved and an oocyte was not obtained. This patient was therefore excluded from the study. No statistically significant differences were noted in patient demographics (age, BMI, baseline endocrine profile (FSH, LH, and $\mathrm{E}_{2}$ ), and ethnicity), day of UDOR, follicular aspirate volume, oocyte maturity, and cycle outcome between the two groups (Table 1).

3.2. Follicular Fluid and Plasma Cytokines. Most FF cytokines (29 out of 40) were found to be at higher concentrations in the MNC group compared to the $\mathrm{COH}$ group (binomial test: $P<0.001)$. This relationship was statistically significant for $\operatorname{LIF}(P<0.01)$ and SDF-1 $\alpha(P<0.05)$ (Figure 1$)$. As with FF, the majority of circulatory cytokines in the MNC cohort were present at higher concentrations than in the $\mathrm{COH}$ group (Figure 2), a relationship which was significant for 12 of these: IL-2ra, IL-3, IL-12 (p40), LIF, M-CSF, IFN$\alpha$, TRAIL, NGF- $\beta$, GRO- $\alpha$, MCP-3, RANTES, and SDF- $\alpha$ $(P<0.05)$. Conversely, plasma IL-12 (p70) levels were present at significantly higher levels following $\mathrm{COH}(P<0.05)$ (Figure 2).

Heat maps were generated following correlation analysis using Kendall's tau to demonstrate FF cytokine interrelationships (Figure 3). Significantly more pairs of cytokines exhibited strong correlations in the MNC data compared to the $\mathrm{COH}$ data (binomial test) $(P<0.001)$. Various relationship alterations were also noted; for example, LIF and TNF- $\alpha$ demonstrated a weak negative correlation in the MNC group (Kendall's tau: -0.08 ) compared with a strong positive correlation following $\mathrm{COH}$ (Kendall's tau: 0.46). When plasma:FF cytokine ratios were compared between the MNC and $\mathrm{COH}$ cohorts, no statistically significant differences were identified.

Gonadotropin use in $\mathrm{COH}$ has previously been recognised to perturb the intrauterine cytokine milieu. Significantly higher concentrations of various cytokines including IL-1 $\beta$, IL-5, IL-10, IL-12, IL-17, TNF- $\alpha$, and eotaxin have been recorded in endometrial secretions from stimulated cycles compared to NCs [68]. Similarly, in the ovary, it has been suggested that exogenous gonadotropins may influence the levels of cytokines such as IL- $1 \beta$, IL- 6 , and TNF- $\alpha$ following earlier studies on FF [69]. More recently, de Los Santos et al. [70] demonstrated altered cumulus cell gene expression for leukocyte differentiation and T-cell activation and regulation following $\mathrm{COH}$, which may in turn influence follicular cytokine profiles as highlighted by the present findings.

To the best of our knowledge, FF has not previously been analysed for such an extensive range of cytokines in $\mathrm{NC} / \mathrm{MNC}$ and $\mathrm{COH}$ cycles. In the present pilot study, FF concentrations of 29 out of the 40 cytokines analysed were found to be higher in the MNC cohort, significantly so for LIF and SDF- $1 \alpha$. LIF is an embryotrophic cytokine whose secretion by GCs and stromal cells into FF has previously 

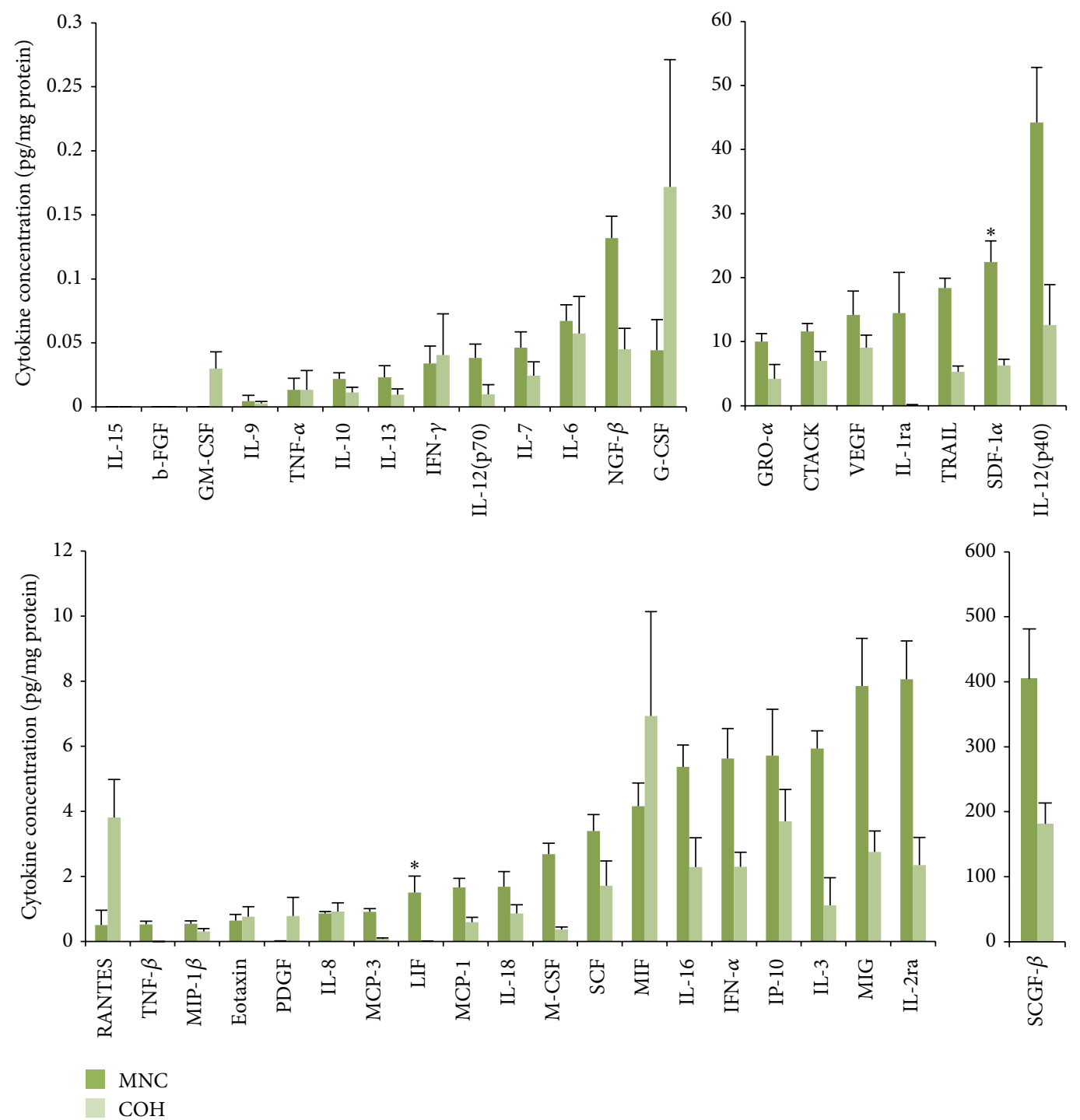

Figure 1: Mean $\left( \pm\right.$ SEM) FF cytokine concentrations in MNC $(n=9)$ and $\mathrm{COH}(n=10)$ cycles $\left({ }^{*}\right.$ significant difference from COH cycle; $P<0.05)$.

TABLE 1: Participant and cycle demographics in MNC and COH cycles.

\begin{tabular}{lccc}
\hline & NC Cycle & COH Cycle & $P$-value \\
\hline Age (years) & $30.8 \pm 0.72(27-34)$ & $31.9 \pm 1.20(24-35)$ & 0.21 \\
BMI $\left(\mathrm{kg} / \mathrm{m}^{2}\right)$ & $23.5 \pm 0.86(20.0-28.0)$ & $24.0 \pm 0.87(20.0-30.0)$ & 0.88 \\
Baseline FSH (IU/L) & $5.2 \pm 0.40(4.0-8.0)$ & $6.0 \pm 0.45(3.9-7.9)$ & 0.24 \\
Baseline LH (IU/L) & $5.2 \pm 0.48(2.6-7.1)$ & $117.8 \pm 18.14(25-193)$ & 0.44 \\
Baseline E $(\mathrm{pmol} / \mathrm{L})$ & $115.2 \pm 12.23(73-162)$ & $13.1 \pm 0.34(12-15)$ & 0.92 \\
Day of cycle for aspiration & $14 \pm 0.93(10-20)$ & $3.0 \pm 0.51(1.0-6.5)$ & 0.35 \\
Follicular volume (mL) & $2.3 \pm 0.40(0.5-4.0)$ & $100^{\mathrm{b}}$ & 0.62 \\
Mature oocyte $(\%)$ & $70^{\mathrm{a}}$ & $30^{\mathrm{c}}$ & 0.06 \\
Clinical pregnancy $(\%)$ & $20^{\mathrm{c}}$ & 0.61 \\
\hline
\end{tabular}

Mean \pm SEM (range), unless otherwise specified. ${ }^{a}$ One patient spontaneously ovulated, therefore 9 oocytes were retrieved; ${ }^{b}$ Follicles yielding a mature oocyte which was subsequently transferred as an embryo were included in this cohort; ${ }^{c}$ Clinical pregnancy rate calculated per embryos transferred [MNC: 7 embryos transferred; $\mathrm{COH} ; 10$ embryos transferred]. 

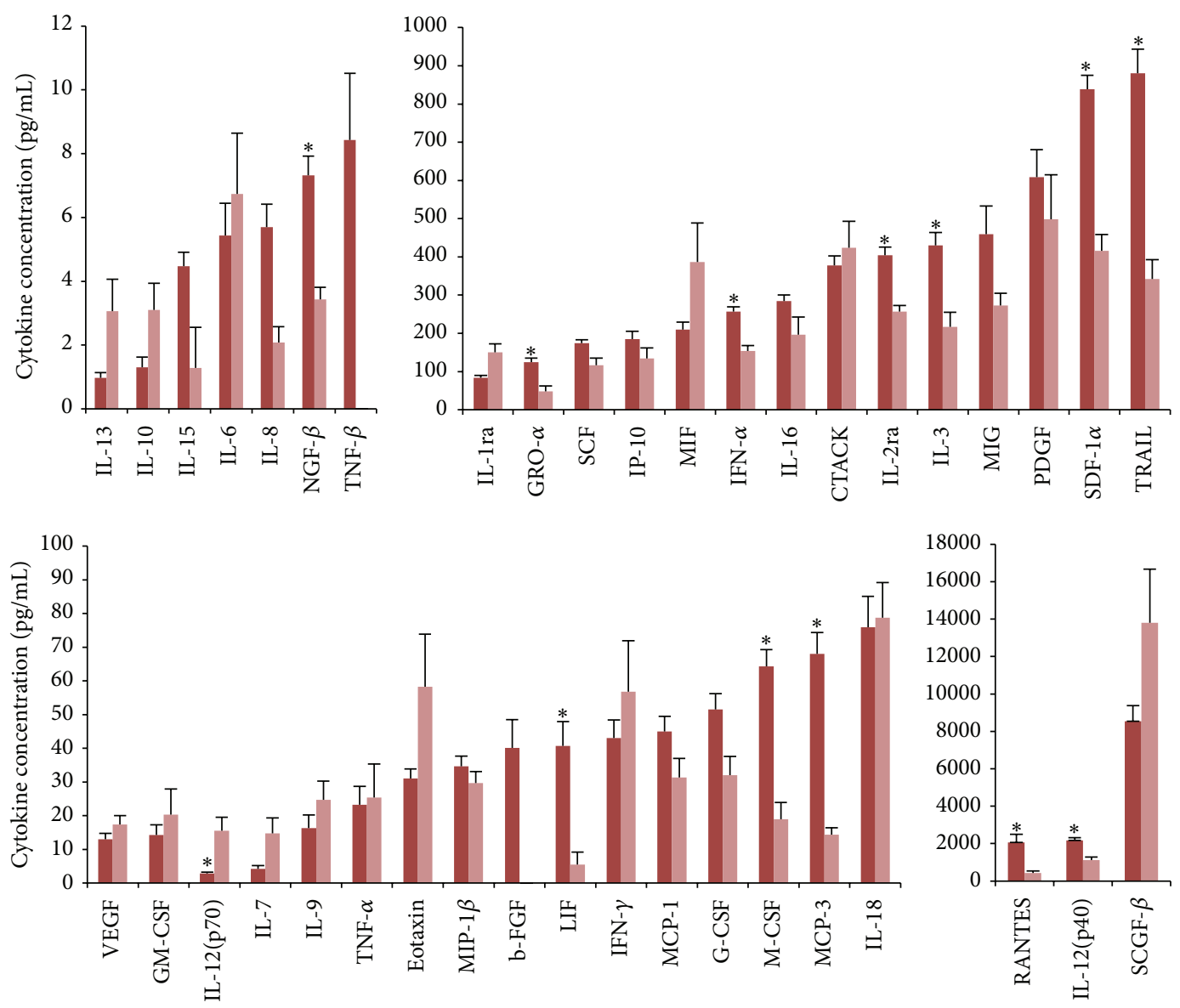

MNC

$\mathrm{COH}$

Figure 2: Mean $\left( \pm\right.$ SEM) plasma cytokine concentrations in MNC $(n=9)$ and $\mathrm{COH}(n=10)$ cycles $\left({ }^{*}\right.$ significant difference from COH cycle; $P<0.05)$.

been shown to be stimulated by hCG [71-73]. Since both the $\mathrm{MNC}$ and $\mathrm{COH}$ cohorts in this study received identical doses of exogenous hCG, the elevated levels of LIF in MNC FF may represent an enhanced response to hCG, whereas the likely perturbed cytokine response allied to $\mathrm{COH}$ may reflect a reduced sensitivity to hCG resulting in lower LIF concentrations. $\mathrm{COH}$ also appeared to perturb relationships between cytokines, highlighted by the relative changes in intrafollicular LIF and TNF- $\alpha$ (where the latter modulates ovarian stromal cell secretion of the former) [71]. The heat map displays a strong positive correlation between LIF and TNF- $\alpha$ in the MNC cohort whereas this relationship is weakened following $\mathrm{COH}$. Interestingly, FF LIF concentrations have also been correlated with $\mathrm{E}_{2}$ concentrations (possibly through its role in enhancing aromatase expression) which, in turn, relate to follicular maturity [74, 75]. Although a comparable causal association has not been identified to date in the ovary, 17- $\beta$ oestradiol is known to induce LIF synthesis in bovine oviduct epithelial cells [76]. It is tempting to speculate that an analogous mechanism is at play in the ovary, where the supraphysiological $\mathrm{E}_{2}$ levels associated with
$\mathrm{COH}$ may impair the induction of follicular LIF synthesis, with a consequent impact on the FF milieu and oocyte quality.

SDF- $1 \alpha$ is a chemokine secreted by oocytes, which acts in a paracrine manner to prevent follicular activation, thereby controlling the entry of primordial follicles into the growing pool in NCs [77]. Furthermore, FF SDF-1 $\alpha$ has previously been positively correlated with FF VEGF levels in $\mathrm{COH}$ cycles, where it is believed to play a proangiogenic role in supporting follicular growth [78]. Our findings corroborate this correlation in the $\mathrm{COH}$ group (Figure 3 ). By contrast, this relationship was much weaker in the MNC cohort, suggesting that it may thus in part be gonadotropin dependent.

Akin to what was noted for FF, plasma cytokine levels in the MNC group were higher than in $\mathrm{COH}$ cycles. The exception was circulatory IL-12 (p70), which was measured at significantly higher concentrations following $\mathrm{COH}$. Furthermore, correlations between FF IL-12 (p70) and other cytokines were markedly altered following stimulation. In the MNC cohort, most FF cytokines were positively correlated with IL-12 (p70), whilst this relationship was reversed following COH. Conversely, IFN- $\gamma$ and TNF- $\alpha$ were negatively 


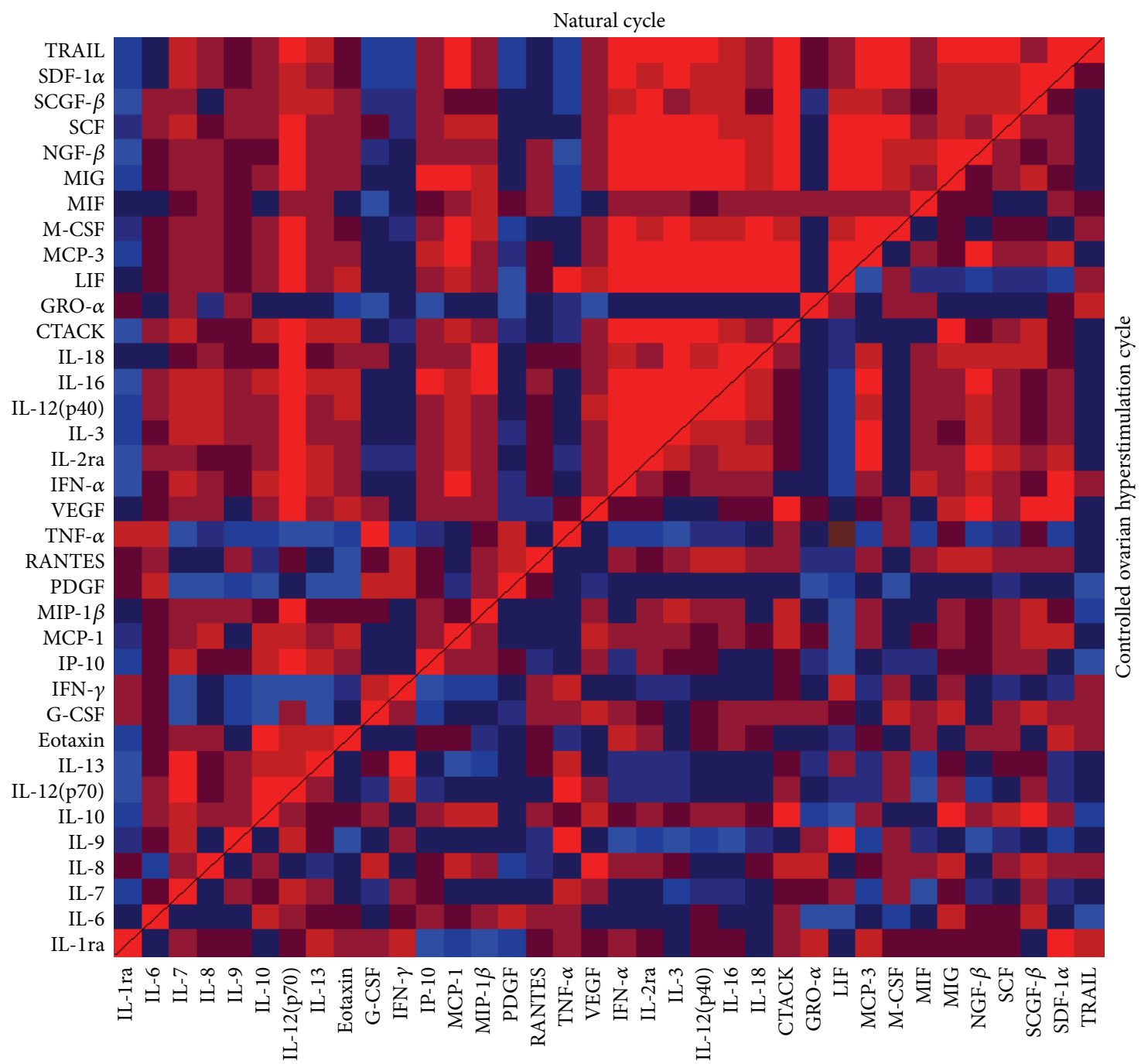

FIGURE 3: Heat maps demonstrating correlations between cytokines in periovulatory FF in MNC and COH cycles. Each square represents the correlation between specific cytokines: a red square represents a significantly positive correlation whilst a blue square represents a significantly negative correlation. A black square represents no significant relationship and the darker shades of red and blue represent weaker positive and negative correlations, respectively.

correlated with IL-12 (p70) in the MNC group and positively in the $\mathrm{COH}$ cohort. Interestingly, an intricate triumvirate relationship between these particular cytokines has been identified in the regulation of inflammatory responses. IL12 (p70) induces IFN- $\gamma$ production [79] and, in turn, IFN$\gamma$ markedly augments IL-12 (p70) production, thereby providing a key inflammatory amplifying mechanism [80]. By contrast, TNF- $\alpha$ is thought to inhibit IFN- $\gamma$-induced IL-12 (p70) production, as demonstrated by Hodge-Dufour et al. [81], whose $\mathrm{TNF}^{+/+}$mice exhibited a prompt inflammatory response which resolved spontaneously compared with a delayed, more vigorous, inflammatory response leading to death associated with elevated IL-12 levels in $\mathrm{TNF}^{-/-}$mice when injected with Corynebacterium parvum. Thus, TNF- $\alpha$ is thought to contribute to the resolution of IL-12- (p70-) driven inflammatory processes. In the MNC cohort, TNF- $\alpha$ was negatively correlated with both IL-12 (p70) and IFN- $\gamma$, thus suggesting that, in the absence of stimulatory gonadotropins, TNF- $\alpha$ of follicular cell origin may play an analogous role via its capacity to regulate IL-12 (p70) production. A positive correlation between these FF cytokines in combination with significantly elevated systemic IL-12 (p70) levels suggests a disruption of this mechanism following $\mathrm{COH}$. An analogous disruption in the ovary following $\mathrm{COH}$ could thus contribute to the detrimental sequelae of increased IL-12 (p70) and IFN- $\gamma$ levels despite the observed compensatory rise in modulatory TNF- $\alpha$ levels.

Fluctuations in systemic white cell populations have been attributed to stimulation with exogenous gonadotropins such that the total number of circulatory leukocytes and neutrophils was increased on the day of hCG administration in $\mathrm{COH}$ compared to NCs [82]. Other studies have reported an increase in plasma white cell count and G-CSF, but not in M-CSF or IL-6, during $\mathrm{COH}$ [83]. Furthermore, various 
cytokines have been found to be elevated in FF following $\mathrm{COH}$, including IL-1 $\beta$, IL-6, and TNF- $\alpha$ [69]. The main difference between the present study and those cited is our administration of exogenous hCG in the MNC cohort. Both LH and hCG induce IL- $1 \beta$ and TNF- $\alpha$, both of which subsequently upregulate GM-CSF expression [38]. Despite identical doses of hCG being used to trigger ovulation in both groups in the current study, GM-CSF in particular was measured at higher (although not statistically significant) levels following $\mathrm{COH}$ in both $\mathrm{FF}$ and plasma. Furthermore, following $\mathrm{COH}$, there was a trend towards several other proinflammatory cytokines in both FF and plasma to be found at higher concentration, with IL-12 (p70) levels in particular being significantly higher in $\mathrm{COH}$ plasma samples. This provides further evidence that $\mathrm{COH}$ can induce both local and systemic inflammatory network deregulations featuring perturbations in cytokine interrelationships which, speculatively, may potentially also impact oocyte viability and treatment outcome. Unfortunately, the present pilot study was not powered to answer this particular question.

\section{Conclusions}

In conclusion, this investigation demonstrated that $\mathrm{COH}$ not only alters FF cytokine profiles compared to MNCs but also perturbs their circulatory levels and disrupts their interrelationships. Given their central role in orchestrating normal follicular physiology, these changes have the potential to adversely affect follicular function and compromise oocyte viability.

\section{Conflict of Interests}

The authors declare that there is no conflict of interests regarding the publication of this paper.

\section{References}

[1] M. J. Pelinck, A. Hoek, A. H. M. Simons, and M. J. Heineman, "Efficacy of natural cycle IVF: a review of the literature," Human Reproduction Update, vol. 8, no. 2, pp. 129-139, 2002.

[2] M. P. Rosen, S. Shen, A. T. Dobson, P. F. Rinaudo, C. E. McCulloch, and M. I. Cedars, "A quantitative assessment of follicle size on oocyte developmental competence," Fertility and Sterility, vol. 90, no. 3, pp. 684-690, 2008.

[3] S. X. Y. Wang, "The past, present, and future of embryo selection in in vitro fertilization: frontiers in reproduction conference," The Yale Journal of Biology and Medicine, vol. 84, no. 4, pp. 487490, 2011.

[4] N. S. Macklon, R. L. Stouffer, L. C. Giudice, and B. C. J. M. Fauser, "The science behind 25 years of ovarian stimulation for in vitro fertilization," Endocrine Reviews, vol. 27, no. 2, pp. 170207, 2006.

[5] J. P. Geraedts and L. Gianaroli, "Embryo selection and IVF," Human Reproduction, vol. 27, no. 9, pp. 2876-2877, 2012.

[6] E. H. Y. Ng, E. Y. L. Lau, W. S. B. Yeung, and P. C. Ho, "Oocyte and embryo quality in patients with excessive ovarian response during in vitro fertilization treatment," Journal of Assisted Reproduction and Genetics, vol. 20, no. 5, pp. 186-191, 2003.
[7] V. Sharma, J. Williams, W. Collins, A. Riddle, B. Mason, and M. Whitehead, "A comparison of treatments with exogenous FSH to promote folliculogenesis in patients with quiescent ovaries due to the continued administration of an LH-RH agonist," Human Reproduction, vol. 2, no. 7, pp. 553-556, 1987.

[8] V. Sharma, M. Whitehead, B. Mason et al., "Influence of superovulation on endometrial and embryonic development," Fertility and Sterility, vol. 53, no. 5, pp. 822-829, 1990.

[9] J. A. Horcajadas, A. Riesewijk, J. Polman et al., "Effect of controlled ovarian hyperstimulation in IVF on endometrial gene expression profiles," Molecular Human Reproduction, vol. 11, no. 3, pp. 195-205, 2005.

[10] E. B. Baart, E. Martini, M. J. Eijkemans et al., "Milder ovarian stimulation for in-vitro fertilization reduces aneuploidy in the human preimplantation embryo: a randomized controlled trial," Human Reproduction, vol. 22, no. 4, pp. 980-988, 2007.

[11] J. A. Horcajadas, P. Díaz-Gimeno, A. Pellicer, and C. Simón, "Uterine receptivity and the ramifications of ovarian stimulation on endometrial function," Seminars in Reproductive Medicine, vol. 25, no. 6, pp. 454-460, 2007.

[12] J. A. Horcajadas, P. Mínguez, J. Dopazo et al., "Controlled ovarian stimulation induces a functional genomic delay of the endometrium with potential clinical implications," Journal of Clinical Endocrinology and Metabolism, vol. 93, no. 11, pp. 45004510, 2008

[13] M. A. Santos, E. W. Kuijk, and N. S. Macklon, "The impact of ovarian stimulation for IVF on the developing embryo," Reproduction, vol. 139, no. 1, pp. 23-34, 2010.

[14] A. Pellicer, A. Ruiz, R. M. Castellvi et al., "Is the retrieval of high numbers of oocytes desirable in patients treated with gonadotrophin-releasing hormone analogues (GnRHa) and gonadotrophins?" Human Reproduction, vol. 4, no. 5, pp. 536$540,1989$.

[15] M. H. van der Gaast, M. J. C. Eijkemans, J. B. van der Net et al., "Optimum number of oocytes for a successful first IVF treatment cycle," Reproductive BioMedicine Online, vol. 13, pp. 476-480, 2006.

[16] M. A. Aboulghar, A. M. Ramzy, R. T. Mansour, Y. M. Amin, and G. I. Serour, "Oocyte quality in patients with severe ovarian hyperstimulation syndrome," Fertility and Sterility, vol. 68, no. 6, pp. 1017-1021, 1997.

[17] G. Ertzeid and R. Storeng, "Adverse effects of gonadotrophin treatment on pre- and postimplantation development in mice," Journal of Reproduction and Fertility, vol. 96, no. 2, pp. 649-655, 1992.

[18] I. Van Der Auwera and T. D'Hooghe, "Superovulation of female mice delays embryonic and fetal development," Human Reproduction, vol. 16, no. 6, pp. 1237-1243, 2001.

[19] C. A. Hodges, A. Ilagan, D. Jennings, R. Keri, J. Nilson, and P. A. Hunt, "Experimental evidence that changes in oocyte growth influence meiotic chromosome segregation," Human Reproduction, vol. 17, no. 5, pp. 1171-1180, 2002.

[20] Y. Li, H.-L. Feng, Y.-J. Cao et al., "Confocal microscopic analysis of the spindle and chromosome configurations of human oocytes matured in vitro," Fertility and Sterility, vol. 85, no. 4, pp. 827-832, 2006.

[21] M. Von Wolff, Z. Kollmann, C. E. Flück et al., "Gonadotrophin stimulation for in vitro fertilization significantly alters the hormone milieu in follicular fluid: a comparative study between natural cycle IVF and conventional IVF," Human Reproduction, vol. 29, no. 5, pp. 1049-1057, 2014. 
[22] A. N. Andersen, P. Devroey, and J.-C. Arce, "Clinical outcome following stimulation with highly purified hMG or recombinant FSH in patients undergoing IVF: a randomized assessor-blind controlled trial," Human Reproduction, vol. 21, no. 12, pp. 32173227, 2006.

[23] A. Weghofer, S. Munné, W. Brannath et al., "The impact of LH-containing gonadotropin stimulation on euploidy rates in preimplantation embryos: antagonist cycles," Fertility and Sterility, vol. 92, no. 3, pp. 937-942, 2009.

[24] L. Regan, E. J. Owen, and H. S. Jacobs, "Hypersecretion of luteinising hormone, infertility, and miscarriage," The Lancet, vol. 336, no. 8724, pp. 1141-1144, 1990.

[25] J. N. Hugues, J. Soussis, I. Calderon et al., "Does the addition of recombinant $\mathrm{LH}$ in WHO group II anovulatory women overresponding to FSH treatment reduce the number of developing follicles? A dose-finding study," Human Reproduction, vol. 20, no. 3, pp. 629-635, 2005.

[26] Z. Shoham, "The clinical therapeutic window for luteinizing hormone in controlled ovarian stimulation," Fertility and Sterility, vol. 77, no. 6, pp. 1170-1177, 2002.

[27] M. Schimberni, F. Morgia, J. Colabianchi et al., "Natural-cycle in vitro fertilization in poor responder patients: a survey of 500 consecutive cycles," Fertility and Sterility, vol. 92, no. 4, pp. 12971301, 2009.

[28] K.-G. Nygren, "Single embryo transfer: the role of natural cycle/minimal stimulation IVF in the future," Reproductive BioMedicine Online, vol. 14, no. 5, article 2841, 2007.

[29] R. Cutting, D. Morroll, S. Roberts, S. Pickering, and A. Rutherford, "Elective single embryo transfer: guidelines for practice British fertility society and association of clinical embryologists," Human Fertility, vol. 11, no. 3, pp. 131-146, 2008.

[30] Joint SOGC-CFAS, "Guidelines for the number of embryos to transfer following in vitro fertilization no. 182, September 2006," International Journal of Gynecology \& Obstetrics, vol. 102, pp. 203-216, 2008.

[31] J. D. Gordon, M. Dimattina, A. Reh, A. Botes, G. Celia, and M. Payson, "Utilization and success rates of unstimulated in vitro fertilization in the United States: an analysis of the Society for Assisted Reproductive Technology database," Fertility and Sterility, vol. 100, no. 2, pp. 392-395, 2013.

[32] B. C. Fauser, G. Nargund, A. N. Andersen et al., "Mild ovarian stimulation for IVF: 10 years later," Human Reproduction, vol. 25, no. 11, pp. 2678-2684, 2010.

[33] G. Nargund, B. C. J. M. Fauser, N. Macklon, W. Ombelet, K. Nygren, and R. Frydman, "The ISMAAR proposal on terminology for ovarian stimulation for IVF," Human Reproduction, vol. 22, no. 11, pp. 2801-2804, 2007.

[34] W. M. Enien, S. El Sahwy, C. P. Harris, M. W. Seif, and M. Elstein, "Human chorionic gonadotrophin and steroid concentrations in follicular fluid: the relationship to oocyte maturity and fertilization rates in stimulated and natural in-vitro fertilization cycles," Human Reproduction, vol. 10, no. 11, pp. 2840-2844, 1995.

[35] M. J. De Los Santos, V. Garca-Lez, D. Beltrn-Torregrosa et al., "Hormonal and molecular characterization of follicular fluid, cumulus cells and oocytes from pre-ovulatory follicles in stimulated and unstimulated cycles," Human Reproduction, vol. 27, no. 6, pp. 1596-1605, 2012.

[36] D. Vinatier, C. Lefebvre-Maunoury, and C. Bernardi, "The ovaries, the immune system, cytokines: physiology," Journal de Gynecologie Obstetrique et Biologie de la Reproduction, vol. 22, no. 6, pp. 581-591, 1993.
[37] C. L. Best and J. A. Hill, "Interleukin- $1 \alpha$ and $-\beta$ modulation of luteinized human granulosa cell oestrogen and progesterone biosynthesis," Human Reproduction, vol. 10, no. 12, pp. 32063210, 1995.

[38] V. Machelon and D. Emilie, "Production of ovarian cytokines and their role in ovulation in the mammalian ovary," European Cytokine Network, vol. 8, no. 2, pp. 137-143, 1997.

[39] D. F. Albertini, C. M. H. Combelles, E. Benecchi, and M. J. Carabatsos, "Cellular basis for paracrine regulation of ovarian follicle development," Reproduction, vol. 121, no. 5, pp. 647-653, 2001.

[40] J. J. Eppig, "Oocyte control of ovarian follicular development and function in mammals," Reproduction, vol. 122, no. 6, pp. 829-838, 2001.

[41] M. K. Skinner, "Regulation of primordial follicle assembly and development," Human Reproduction Update, vol. 11, no. 5, pp. 461-471, 2005.

[42] S. Vujisić, S. Ž. Lepej, I. Emedi, R. Bauman, A. Remenar, and M. K. Tiljak, "Ovarian follicular concentration of IL-12, IL-15, IL-18 and p40 subunit of IL-12 and IL-23," Human Reproduction, vol. 21, no. 10, pp. 2650-2655, 2006.

[43] S. Vujisic and S. Zidovec, "Follicular immunology environment and the influence on in-vitro fertilization outcome," Current Women's Health Reviews, vol. 1, pp. 49-60, 2005.

[44] C. Mendoza, N. Cremades, E. Ruiz-Requena et al., "Relationship between fertilization results after intracytoplasmic sperm injection, and intrafollicular steroid, pituitary hormone and cytokine concentrations," Human Reproduction, vol. 14, no. 3, pp. 628-635, 1999.

[45] M. E. Hammadeh, A. K. Ertan, M. Baltes et al., "Immunoglobulins and cytokines level in follicular fluid in relation to etiology of infertility and their relevance to IVF outcome," American Journal of Reproductive Immunology, vol. 47, no. 2, pp. 82-90, 2002.

[46] C. Mendoza, E. Ruiz-Requena, E. Ortega et al., "Follicular fluid markers of oocyte developmental potential," Human Reproduction, vol. 17, no. 4, pp. 1017-1022, 2002.

[47] M. E. Hammadeh, C. Fischer-Hammadeh, A. S. Amer, P. Rosenbaum, and W. Schmidt, "Relationship between cytokine concentration in serum and preovulatory follicular fluid and in vitro fertilization/intracytoplasmic sperm injection outcome," Chemical Immunology and Allergy, vol. 88, pp. 80-97, 2005.

[48] M. Bedaiwy, A. Y. Shahin, A. M. AbulHassan et al., "Differential expression of follicular fluid cytokines: relationship to subsequent pregnancy in IVF cycles," Reproductive BioMedicine Online, vol. 15, no. 3, pp. 321-325, 2007.

[49] N. Lédée, R. Lombroso, L. Lombardelli et al., "Cytokines and chemokines in follicular fluids and potential of the corresponding embryo: the role of granulocyte colony-stimulating factor," Human Reproduction, vol. 23, no. 9, pp. 2001-2009, 2008.

[50] A. Revelli, L. D. Piane, S. Casano, E. Molinari, M. Massobrio, and P. Rinaudo, "Follicular fluid content and oocyte quality: from single biochemical markers to metabolomics," Reproductive Biology and Endocrinology, vol. 7, article 40, 2009.

[51] N. Lédée, M. Petitbarat, M. Rahmati et al., "New pre-conception immune biomarkers for clinical practice: interleukin-18, interleukin-15 and TWEAK on the endometrial side, G-CSF on the follicular side," Journal of Reproductive Immunology, vol. 88, no. 2, pp. 118-123, 2011.

[52] A. Sarapik, A. Velthut, K. Haller-Kikkatalo et al., "Follicular proinflammatory cytokines and chemokines as markers of IVF 
success," Clinical and Developmental Immunology, vol. 2012, Article ID 606459, 10 pages, 2012.

[53] H. Hagberg, C. Mallard, and B. Jacobsson, "Role of cytokines in preterm labour and brain injury," British Journal of Obstetrics and Gynaecology, vol. 112, supplement 1, pp. 16-18, 2005.

[54] A. Agic, H. Xu, D. Finas, C. Banz, K. Diedrich, and D. Hornung, "Is endometriosis associated with systemic subclinical inflammation?" Gynecologic and Obstetric Investigation, vol. 62, no. 3, pp. 139-147, 2006.

[55] O. B. Christiansen, H. S. Nielsen, and A. M. Kolte, "Inflammation and miscarriage," Seminars in Fetal and Neonatal Medicine, vol. 11, no. 5, pp. 302-308, 2006.

[56] A. Arici, E. Oral, O. Bukulmez, S. Buradagunta, O. Engin, and D. L. Olive, "Interleukin-8 expression and modulation in human preovulatory follicles and ovarian cells," Endocrinology, vol. 137, no. 9, pp. 3762-3769, 1996.

[57] E. Runesson, K. Ivarsson, P. O. Janson, and M. Brännström, "Gonadotropin- and cytokin-regulated expression of the chemokine interleukin 8 in the human preovulatory follicle of the menstrual cycle," Journal of Clinical Endocrinology and Metabolism, vol. 85, no. 11, pp. 4387-4395, 2000.

[58] V. Baud and M. Karin, "Signal transduction by tumor necrosis factor and its relatives," Trends in Cell Biology, vol. 11, no. 9, pp. 372-377, 2001.

[59] R. P. Numerof and K. Asadullah, "Cytokine and anti-cytokine therapies for psoriasis and atopic dermatitis," BioDrugs, vol. 20, no. 2, pp. 93-103, 2006.

[60] S. Weiser, J. Miu, H. J. Ball, and N. H. Hunt, "Interferon$\gamma$ synergises with tumour necrosis factor and lymphotoxin$\alpha$ to enhance the mRNA and protein expression of adhesion molecules in mouse brain endothelial cells," Cytokine, vol. 37, no. 1, pp. 84-91, 2007.

[61] N. A. Bersinger, Z. Kollmann, and M. von Wolff, "Serum but not follicular fluid cytokine levels are increased in stimulated versus natural cycle IVF: a multiplexed assay study," Journal of Reproductive Immunology, vol. 106, pp. 27-33, 2014.

[62] O. Tokuyama, Y. Nakamura, A. Muso, Y. Fujino, O. Ishiko, and S. Ogita, "Vascular endothelial growth factor concentrations in follicular fluid obtained from IVF-ET patients: a comparison of hMG, clomiphene citrate, and natural cycle," Journal of Assisted Reproduction and Genetics, vol. 19, no. 1, pp. 19-23, 2002.

[63] J. S. Cunha-Filho, N. Lemos, N. Stein, A. Laranjeira, and E. P. Passos, "Vascular endothelial growth factor and inhibin A in follicular fluid of infertile patients who underwent in vitro fertilization with a gonadotropin-releasing hormone antagonist," Fertility and Sterility, vol. 83, no. 4, pp. 902-907, 2005.

[64] C. McRae, N. E. Baskind, N. M. Orsi, V. Sharma, and J. Fisher, "Metabolic profiling of follicular fluid and plasma from natural cycle in vitro fertilization patients-a pilot study," Fertility and Sterility, vol. 98, no. 6, pp. 1449.e6-1457.e6, 2012.

[65] N. M. Orsi, N. Gopichandran, U. V. Ekbote, and J. J. Walker, "Murine serum cytokines throughout the estrous cycle, pregnancy and post partum period," Animal Reproduction Science, vol. 96, no. 1-2, pp. 54-65, 2006.

[66] P. F. Levay, C. Huyser, F. L. Fourie, and D. J. Rossouw, "The detection of blood contamination in human follicular fluid," Journal of Assisted Reproduction and Genetics, vol. 14, no. 4, pp. 212-217, 1997.

[67] C. Huyser, F. L. R. Fourie, and L. Wolmarans, "Spectrophotometric absorbance of follicular fluid: a selection criterion," Journal of Assisted Reproduction and Genetics, vol. 9, no. 6, pp. 539-544, 1992.
[68] C. M. Boomsma, A. Kavelaars, M. J. C. Eijkemans, B. C. J. M. Fauser, C. J. Heijnen, and N. S. MacKlon, "Ovarian stimulation for in vitro fertilization alters the intrauterine cytokine, chemokine, and growth factor milieu encountered by the embryo," Fertility and Sterility, vol. 94, no. 5, pp. 1764-1768, 2010.

[69] J. R. L. de Mola, J. M. Goldfarb, B. R. Hecht, G. P. Baumgardner, C. J. Babbo, and M. A. Friedlander, "Gonadotropins induce the release of interleukin- $1 \beta$, interleukin- 6 and tumor necrosis factor- $\alpha$ from the human preovulatory follicle," American Journal of Reproductive Immunology, vol. 39, no. 6, pp. 387-390, 1998.

[70] M. J. de Los Santos, V. Garca-Lez, D. Beltrn-Torregrosa et al., "Hormonal and molecular characterization of follicular fluid, cumulus cells and oocytes from pre-ovulatory follicles in stimulated and unstimulated cycles," Human Reproduction, vol. 27, no. 6, pp. 1596-1605, 2012.

[71] A. Arici, E. Oral, O. Bahtiyar, O. Engin, E. Seli, and E. E. Jones, "Leukaemia inhibitory factor expression in human follicular fluid and ovarian cells," Human Reproduction, vol. 12, no. 6, pp. 1233-1239, 1997.

[72] S. Coskun, M. Uzumcu, K. Jaroudi, J. M. G. Hollanders, R. S. Parhar, and S. T. Al-Sedairy, "Presence of leukemia inhibitory factor and interleukin-12 in human follicular fluid during follicular growth," The American Journal of Reproductive Immunology, vol. 40, no. 1, pp. 13-18, 1998.

[73] M. Jean, S. Mirallie, P. Barriere et al., "Leukaemia inhibitory factor expression in human follicular fluid," Human Reproduction, vol. 14, no. 2, article 571, 1999.

[74] Y. Zhao, J. E. Nichols, S. E. Bulun, C. R. Mendelson, and E. R. Simpson, "Aromatase P450 gene expression in human adipose tissue: role of a Jak/STAT pathway in regulation of the adiposespecific promoter," The Journal of Biological Chemistry, vol. 270, no. 27, pp. 16449-16457, 1995.

[75] O. Bukulmez and A. Arici, "Leukocytes in ovarian function," Human Reproduction Update, vol. 6, no. 1, pp. 1-15, 2000.

[76] K. C. Reinhart, R. K. Dubey, C. L. Mummery, M. Van Rooijen, P. J. Keller, and R. Marinella, "Synthesis and regulation of leukaemia inhibitory factor in cultured bovine oviduct cells by hormones," Molecular Human Reproduction, vol. 4, no. 3, pp. 301-308, 1998.

[77] J. E. Holt, A. Jackson, S. D. Roman, R. J. Aitken, P. Koopman, and E. A. McLaughlin, "CXCR4/SDF1 interaction inhibits the primordial to primary follicle transition in the neonatal mouse ovary," Developmental Biology, vol. 293, no. 2, pp. 449-460, 2006.

[78] A. Nishigaki, H. Okada, R. Okamoto et al., "Concentrations of stromal cell-derived factor-1 and vascular endothelial growth factor in relation to the diameter of human follicles," Fertility and Sterility, vol. 95, no. 2, pp. 742-746, 2011.

[79] T. Hamza, J. B. Barnett, and B. Li, "Interleukin 12 a key immunoregulatory cytokine in infection applications," International Journal of Molecular Sciences, vol. 11, no. 3, pp. 789-806, 2010.

[80] G. Trinchieri, "Interleukin-12: a proinflammatory cytokine with immunoregulatory functions that bridge innate resistance and antigen-specific adaptive immunity," Annual Review of Immunology, vol. 13, pp. 251-276, 1995.

[81] J. Hodge-Dufour, M. W. Marino, M. R. Horton et al., "Inhibition of interferon $\gamma$ induced interleukin 12 production: a potential 
mechanism for the anti-inflammatory activities of tumor necrosis factor," Proceedings of the National Academy of Sciences of the United States of America, vol. 95, no. 23, pp. 13806-13811, 1998.

[82] A. Giuliani, W. Schoell, J. Auner, and W. Urdl, "Controlled ovarian hyperstimulation in assisted reproduction: effect on the immune system," Fertility and Sterility, vol. 70, no. 5, pp. 831-815, 1998.

[83] A. Hoek, J. Schoemaker, and H. A. Drexhage, "Premature ovarian failure and ovarian autoimmunity," Endocrine Reviews, vol. 18, no. 1, pp. 107-134, 1997. 


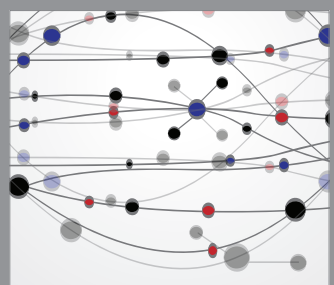

The Scientific World Journal
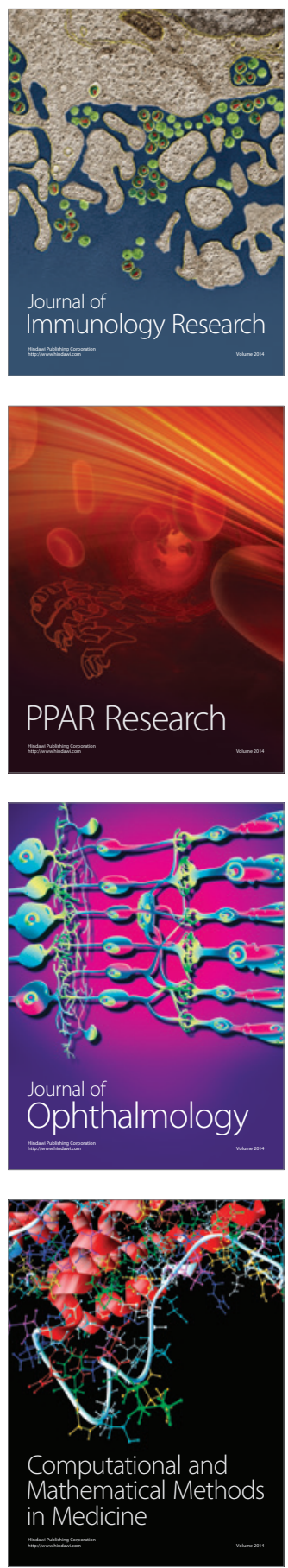

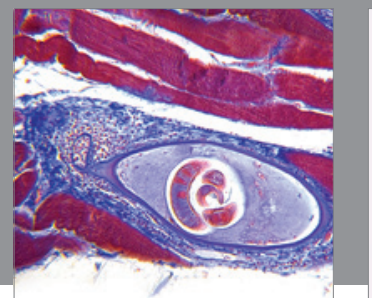

Gastroenterology

Research and Practice
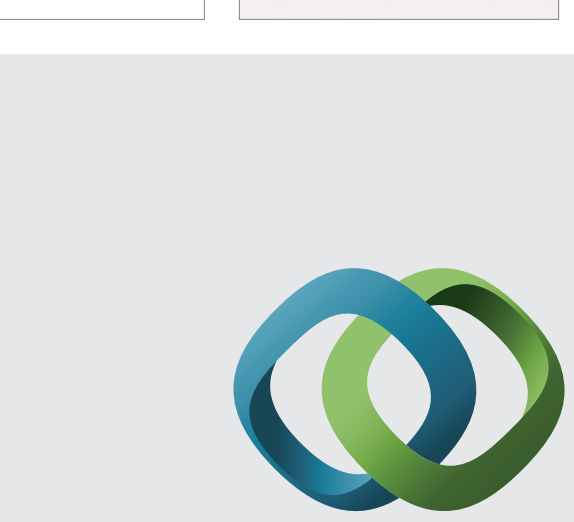

\section{Hindawi}

Submit your manuscripts at

http://www.hindawi.com
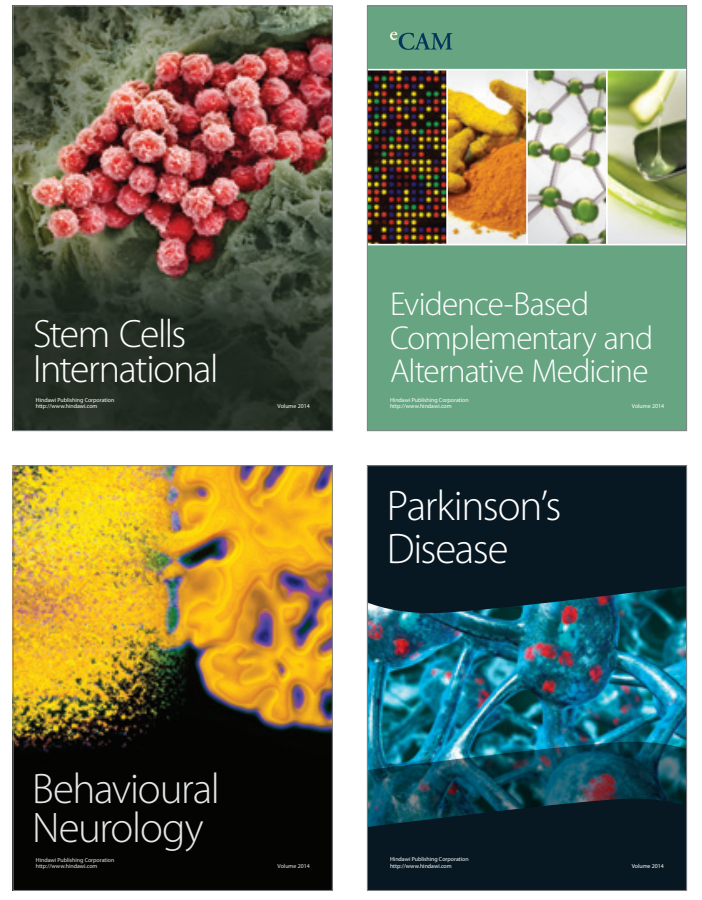
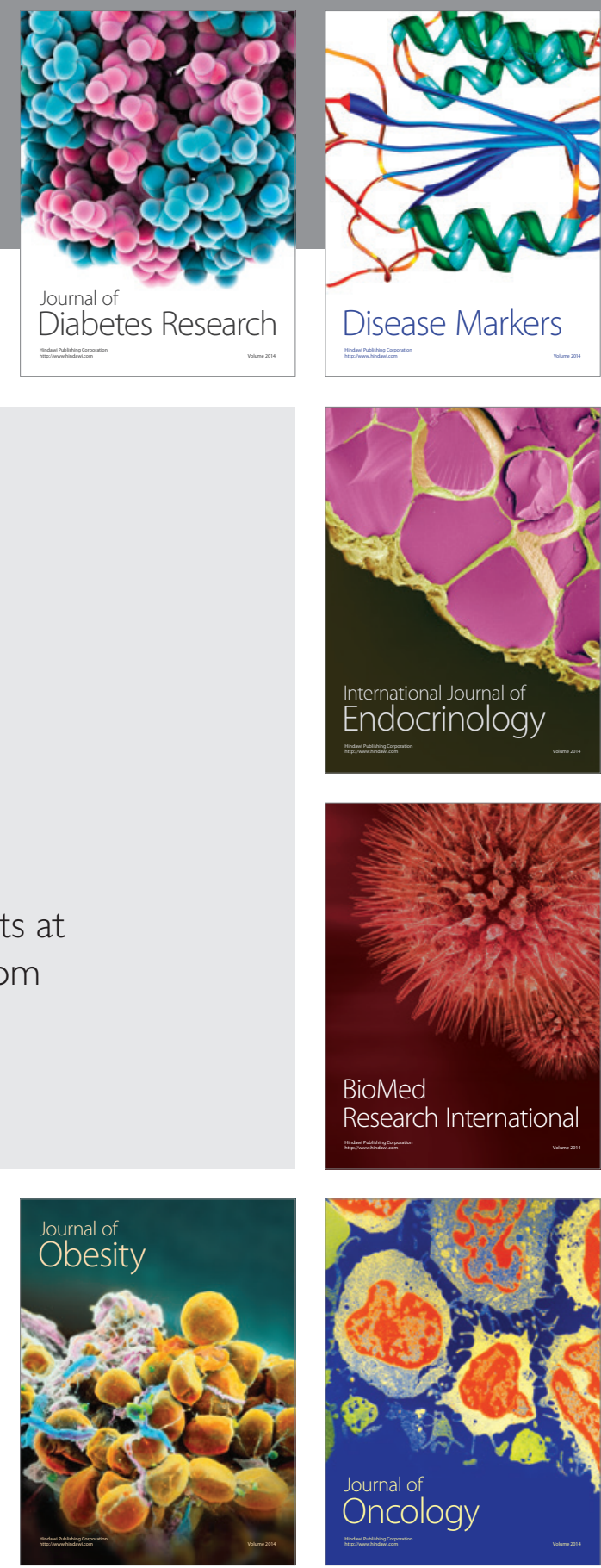

Disease Markers
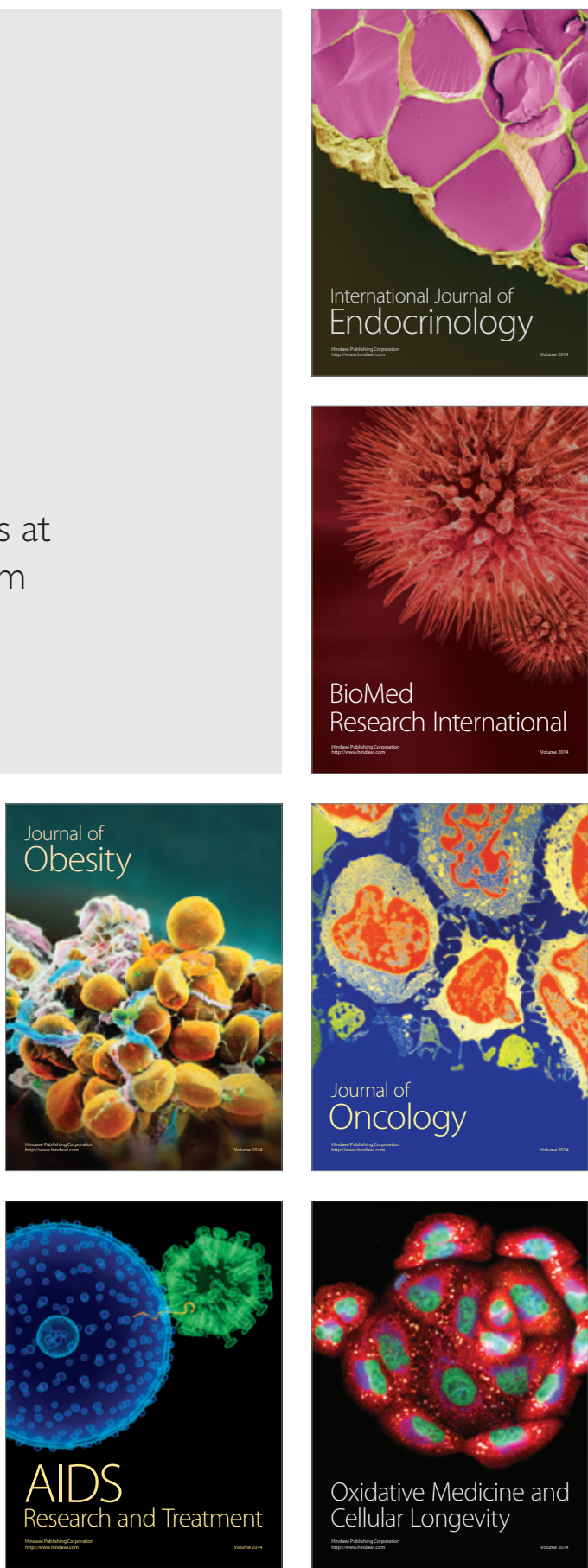